\title{
Vehicle Color Recognition in Static Image For Traffic Enforcement Camera System
}

\author{
Tang Yong ${ }^{1}$ \\ 1. School of Automobile and Traffic Engineering, \\ Nanjing Forestry University, \\ Nanjing, China \\ E-mail: tangyong159@163.com \\ * Corresponding Author
}

\begin{abstract}
Vehicle information recognition is a key component of traffic enforcement camera system. Color plays an important role in vehicle identification. In this paper, we propose a method of vehicle color recognition based on SVM and Bayesian classifier combination. For distinguished vehicle color types, support vector machine algorithms is used for classification. After generating prior probability and class conditional probability, maximum posterior probability is computed based on Bayes classifier to identify color types for less-distinguishable colors type. Then support vector machine and Bayes classifier are combined to with decision tree, which simplified classification algorithms. Our experiment show that a good identification of color types is achieved, and the total recognition rate is at $86.5 \%$.
\end{abstract}

Keywords- Vehicle Color Recognition; Support Vector Machine; Bayes Classifier; Multiple Classifier Combination

\section{INTRODUCTION}

In order to strengthen the public security, many cities started to set up traffic enforcement camera system for monitoring vehicles [1]. When a vehicle passed the road under traffic enforcement camera system, the camera immediately take photos of the vehicle and recorded, as shown in Figure 1. In early days, huge vehicle images had to be absolutely artificially recognized and classified, which was too inefficient and with high human cost. With the development of technology, new technologies such as computer vision and pattern recognition have been applied to such system. For example, automatic license plate recognition technology [2] could achieve automatic recognition of vehicles involved in illegal activities and crimes by computer's automatic recognition of license plates, which included retrieval, check and trajectory tracking [3]. However, there were also defects on detecting and recognizing vehicles simply by license plates, such as blurred shots, stained license plate or fake license plates used by crime vehicles, which would lead to the failure or nullity of license plate recognition [4]. At present, some researchers have begun to resort to computer vision technology to extract more vehicle characteristics, such as vehicle type, vehicle color, vehicle logo, and shape of vehicle headlight, etc. Color is one of the most dominant cues for vehicle identification. Color recognition in natural scenes can provide useful information in vehicle detection vehicle tracking and automatic driving system.

\author{
$\mathrm{Xu}$ Yan \\ 1. School of Automobile and Traffic Engineering, \\ Nanjing Forestry University, \\ Nanjing, China \\ E-mail: 1071048444@qq.com
}

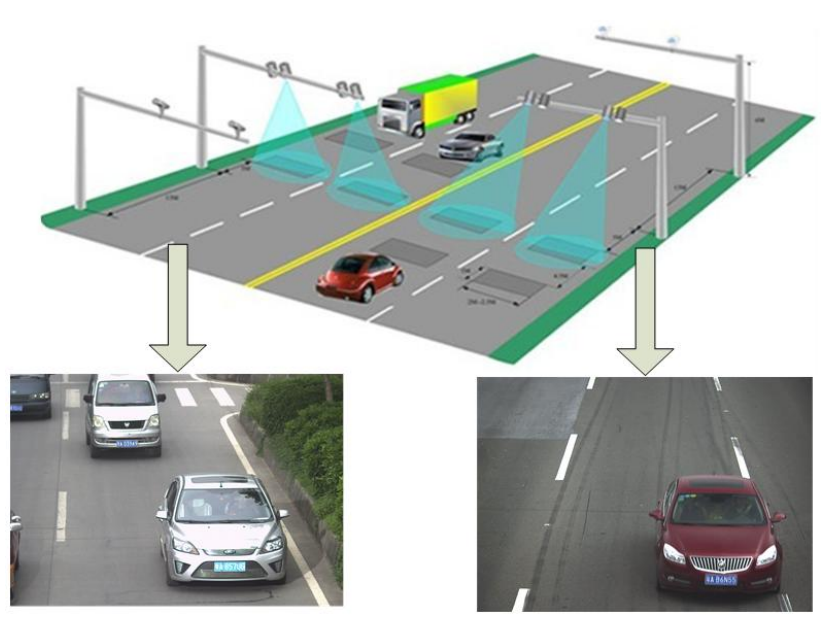

Figure 1. traffic enforcement camera system

Color was a very distinguishing characteristic of vehicles, but it was also apt to be disturbed by external environment factors such as light, weather and camera image sensor devices. Besides, people's subjective psychology and consciousness would also exert an effect on human eye's perception of color. So it is a challenging work to recognize the colors of vehicles body.

In vehicle color recognition field, Researchers proposed a number of method as follow. Wang et al. [5] investigated the multiple support vector machine for feature selection in the classification of lip color. Zheng et al. [6] described a method that using SVM and color histogram for classifying color. Son et al. [7] introduced a convolution kernel that extracted the color information of vehicle body images. Guijun Li et al. [8] achieved high color recognition rate that using the standard color difference formula to recognize vehicle color in relatively HIS color space and. Na Jing et al. [9] made the comparison of recognition algorithms in different color spaces. Kim K.J. et al. [10] proposed a means to recognize vehicle colors by extracting color histogram characteristics.

In this paper, we described a classifier that combined by support vector machine and Bayes classifier with a decision tree for vehicle color recognition, which is then simplified to binary classifier problem. This paper is organized as follows. In Section II, we introduce the details of our algorithm, which includes SVM algorithm and Bayes classifier. The experiment results on images are 
presented and discussed in Section III. Section IV is the conclusion of this paper.

\section{COLOR RECOGNITION ALGORITHM}

\section{A. Color Space Choice}

There are several common color spaces for color recognition, which are $\mathrm{RGB}, \mathrm{HSV}, \mathrm{YCbCr}$ and so on. No best color space for recognition since there is no color space that can satisfy all conditions. In this work we use the HSV color space instead of the RGB color space [11]. Moreover, we use only Hue and Saturation and ignore $\mathrm{V}$ (brightness) in order to minimize the influence of shadow and uneven lighting.

Formula (1) - (3) is used to convert RGB to HSV color space.

$$
\begin{aligned}
& V=\max (R, G, B) \quad \mathrm{V}=0 \\
& S= \begin{cases}\frac{0}{\max -\min } & \mathrm{V}=0 \\
\max & \mathrm{V} \neq 0\end{cases}
\end{aligned}
$$

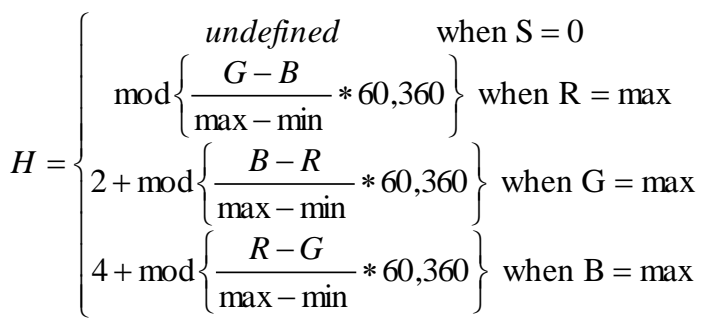

\section{B. Color Classification Based on SVM}

Support vector machine (SVM) is a powerful classification methodology that can create specialized functions from training data set with label [12]. The foundation of support vector machine (SVM) was developed by Vapnik in 1995 [13]. The following Figure 5 was the schematic diagram of two-dimensional linear classification. The middle line was classification hyperplane, which could make the gap margin maximum between separated sample points. The sample vector which was closest to hyperplane was called support vector, and a set of support vectors could uniquely determine one hyperplane.

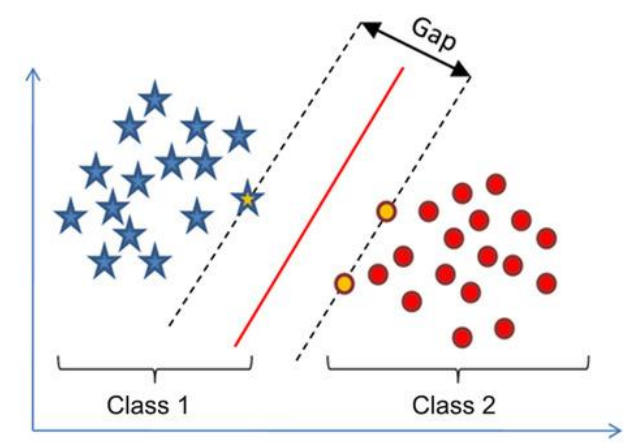

Figure 2. The Binary Classification of Samples

Consider the problem of separating the set of training data belonging to two linearly separable classes: $\left(x_{i}, y_{i}\right), i=1,2, \ldots n, x \in R^{d}, x \in\{+1,-1\}$ with a hyperplane:

$$
w \bullet x+b=0
$$

The set of data is be optimally separated by a hyperplane if it is separated without error. When the data points is nonseparable in linear, a slack variable $\xi_{i}$ could be introduced to transfer computing optimal hyperplane to convex quadratic programming. The constraint conditions were:

$$
y_{i}\left(w \bullet x_{i}+b\right) \geq 1-\xi_{i}
$$

The aim of optimization becomes:

$$
\min \frac{1}{2}\|w\|^{2}+C \sum_{i=1}^{N} \xi_{i}
$$

$\mathrm{C}$ referred to constant value that was called the penalty factor. By using Lagrangian function, the optimization problem in Formula 3 and Formula 4 could be translated as follow:

$$
\begin{aligned}
& \max \left\{\sum_{i=1}^{N} a_{i}-\frac{1}{2} \sum_{i=1}^{n} a_{i} a_{j} w_{i} w_{j} k\left(x_{i}, x_{j}\right)\right\} \\
& \text { s.t. } \quad \sum_{i=1}^{N} a_{i} y_{i}=0
\end{aligned}
$$

$$
a_{i} \geq 0
$$

$a_{i}$ referred to Lagrange Coefficient, and $k\left(x_{i}, y_{j}\right)=x_{i} \bullet y_{i}$ is the kernel function performing the nonlinear mapping into feature space [13].

Combining $\mathrm{H}$ and $\mathrm{S}$ value as feature, we can use SVM algorithm for color classification. Firstly, color values of $\mathrm{H}$ and $\mathrm{S}$ were normalized to $[-1,1]$, and then used radial basis function (6) as kernel function to carry out binary classification.

$$
k\left(x_{i}, y_{j}\right)=\exp \left(-\gamma\left\|x_{i}-y_{i}\right\|^{2}\right)
$$

\section{Color Classification based on Bayesian Classifier}

SVM algorithm was used to classify colors that were clustered well in $\mathrm{H}-\mathrm{S}$ space, but it could not distinguish colors well with high coincidence, such as colors of white, black and gray. Therefore, we applied Bayesian classifier to recognize and distinguish the colors through statistical regularity of $\mathrm{H}$-value. Distribution of $\mathrm{H}$-value for each color mostly meet Gaussian distribution, and the parameter of Gaussian distribution for each color could be calculated by the statistics of histogram. With evaluating the statistics of sample images, we could obtain following information:

(1) Frequency of occurrence of the certain corresponding color class.

(2) Frequency equivalent to the prior probability $p\left(\omega_{i}\right)$ under the condition of a large number of samples

(3) Class-conditional probability of the color type.

According to Bayesian theory [14], the maximum posterior probability $p\left(\omega_{i} \mid X\right)$ of which a sample belonged to a type is as follow:

$$
P\left(\omega_{i} \mid X\right)=\frac{P\left(X \mid \omega_{i}\right) P\left(\omega_{i}\right)}{\sum_{j=1}^{M} P\left(X \mid \omega_{i}\right) P\left(\omega_{i}\right)}
$$

$P\left(\omega_{i} \mid X\right)$ referred to the probability of which samples was to be belonged to the class $\omega_{i}$. Taken recognizing the color of black as example, the classification problem is simplified as the black and non-black. Black class was set as, non-black class was set as $\bar{w}_{b}$, character vector of samples to be detected was $\mathrm{H}$ and Bayesian classifier was: 


$$
\operatorname{Pr}\left(w_{b} \mid H\right)=\frac{\operatorname{Pr}\left(H \mid w_{b}\right) \operatorname{Pr}\left(w_{b}\right)}{\operatorname{Pr}\left(H \mid w_{b}\right) \operatorname{Pr}\left(w_{b}\right)+\operatorname{Pr}\left(H \mid \bar{w}_{b}\right) \operatorname{Pr}\left(\bar{w}_{b}\right)}
$$

$\operatorname{Pr}\left(w_{b} \mid H\right)$ referred to the probability that samples to be detected belonged to $\operatorname{Pr}\left(H \mid w_{b}\right)$ and $\operatorname{Pr}\left(H \mid \bar{w}_{b}\right)$ respectively referred to the probability of character vectors in all the class and $\bar{w}_{b}$ class, which could be gained by using histogram for the statistics of training samples. $\operatorname{Pr}\left(\bar{w}_{b}\right)$ respectively referred to the proportion of total number of black samples (positive samples) and non-black samples (negative samples) in training samples (equivalent to the prior probability under the condition of a large number of samples). The ratio in Formula (9) was used to determine the sample classification.

$$
K_{w_{b}}=\frac{\operatorname{Pr}\left(w_{b} \mid H\right)}{\operatorname{Pr}\left(\bar{w}_{b} \mid H\right)}>\theta_{w_{b}}
$$

In order to determine $\theta_{w_{b}}$, we normalized $K_{w_{b}} \in[0,+\infty)$ to $K_{w_{b}}^{\text {norm }} \in[0,1]$. In order to dynamically adjust the distribution scope of, we could compress the interval of a smaller distribution probability and extend the region of larger distribution probability to make it as $[0,1]$ by probability distribution equilibrium normalization method [14], and the judgment criterion was revised as $K_{w_{b}}^{n o r m}>\theta_{v_{b}}^{n o r m}$. The value of $\theta_{w_{b}}^{\text {norm }}$ was determined by making a synthetic comparison of false detection rate and missing rate with the training. In order to optimize the value of $\theta_{w_{b}}^{\text {norm }}$, the false detection rate $R_{E}$ and miss rate $R_{M}$ in classification detection were defined respectively as follows:

$$
\begin{aligned}
& R_{E}=\frac{N_{E}}{N_{E}+N_{D}} \times 100 \% \\
& R_{M}=\frac{N_{M}}{N_{M}+N_{D}} \times 100 \%
\end{aligned}
$$

$N_{E}$ referred to the detected number of positive samples, namely the number of negative samples that were mistakenly recognized as positive samples. $N_{D}$ referred to the detected number of positive samples and $N_{M}$ was the undetected number of black samples. When $\theta_{w_{b}}^{\text {norm }}$ varied, false detection rate decreased as threshold increased and miss rate increased as threshold increased. When false detection rate and missing rate were in balance (nearly equal), the value of $\theta_{w_{b}}^{n o r m}$ was considered as the optimal threshold.

\section{Combination of Multiple Classifiers}

SVM algorithm could only carry out binary classification of samples while there were multiclassification problems in actual practice, just like the color recognition in our research. To effectively classify and recognize colors, this study set up a decision tree based on characteristics of colors to gradually convert multi-classification problems to two-class classification problems, which indicated that several two-class classifiers composed a multiple classifier to improve the speed and precision of detection. We could set up a decision tree by
SVM and Bayesian classifier and make judgments on the top of the decision tree as for colors that were easily distinguished, as shown in Figure 3. For example, green and yellow could be quickly recognized and eliminated by using SVM. As for colors that were difficult to detect, we could make judgments at the bottom of the decision tree. Thus, in the process of detection, we could quickly rule out a large number of colors that were easy to distinguish to accelerate the detection speed. On the other hand, the combination of Bayesian classifiers and SVM classifiers also helped realize algorithm programming.

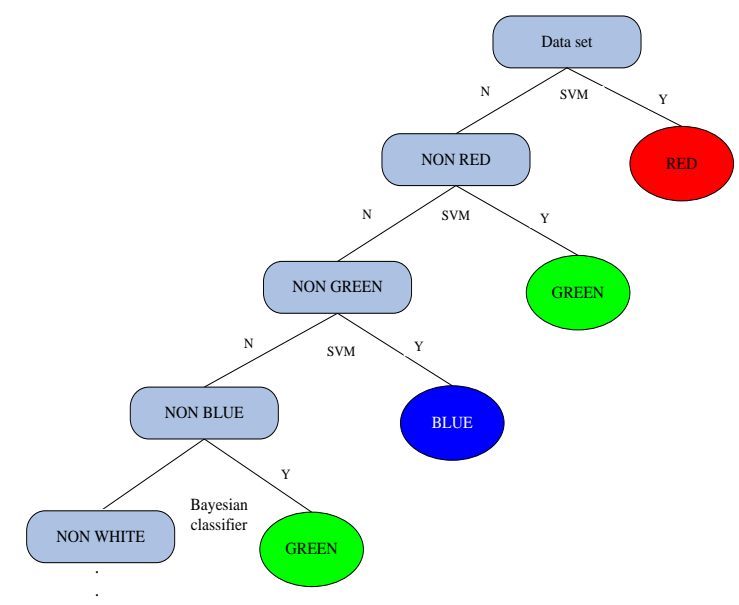

Figure 3. Multi-Color Recognition Based on The Combination of Multiple Classifiers

\section{EXPERIMENTS}

This section will introduce the results of the vehicle color recognition testing. Our results are all calculated on a desktop computer with an Intel Core i7, 3.4GHz CPU, and 4GB RAM. The software development platform was Visual Studio 2005 and OpenCV 2.4.3.

\section{A. Date Set for Training}

The local police department in Maanshan City provided a large collection of vehicle images recorded with traffic surveillance cameras in one week. The capturing time was between 7:00 (AM) and 17:00 (PM) with a wide range of illumination condition. Several type of vehicle were collected for our testing as follow. The percentage of color sample accounting for total number of samples was set as the prior probability of the color class. The quantity of vehicle color samples and statistical regularity is in Table 1.

TABLE I. STATISTIC OF TRAINING SAMPLES

\begin{tabular}{|c|c|c|c|c|c|c|c|}
\hline & black & white & gray & red & blue & green & yellow \\
\hline number & 1781 & 855 & 445 & 341 & 131 & 96 & 11 \\
\hline percent & $48 \%$ & $23 \%$ & $12 \%$ & $9.3 \%$ & $3.6 \%$ & $2.7 \%$ & $0.3 \%$ \\
\hline
\end{tabular}

\section{B. Results of Recognition}

Since there were few green and yellow vehicles in the images taken at the traffic monitoring system (less than ten), the experimental result was not representative, meanwhile the study did not detect the two colors. The final recognition result of several vehicle colors was shown in Table 2. 
TABLE II. Statistic ResUlts OF COLOR RECOGNITION

\begin{tabular}{|c|c|c|c|c|c|c|}
\hline & black & white & gray & red & blue & total \\
\hline number & 147 & 121 & 93 & 137 & 23 & 521 \\
\hline $\begin{array}{c}\text { correct } \\
\text { number }\end{array}$ & 130 & 105 & 70 & 125 & 21 & 451 \\
\hline $\begin{array}{c}\text { correct } \\
\text { rate }\end{array}$ & $88.4 \%$ & $86.7 \%$ & $75.2 \%$ & $91.2 \%$ & $91.3 \%$ & $86.5 \%$ \\
\hline
\end{tabular}

According to Table 2, several colors that were better clustered by using SVM algorithm realized a higher recognition rate while recognition rate for gray vehicles was low, which was related to the poor color clustering performance. The total recognition rate for vehicles of all colors reached $86.5 \%$. Batch inspection of all the 521 images cost about 50 seconds and each image cost less than 100 milliseconds. The efficiency was pretty high.

\section{CONCLUSIONS}

Vehicle color recognition was an important auxiliary means of the vehicle recognition system, playing an important role in traffic investigation, traffic management and vehicle management system. In this paper, an effective method using SVM and Bayesian classifier for vehicle color recognition has been proposed. However, due to the subjectivity in the process of color classification and the complexity of vehicle colors, for example, vehicle color would change with the variation of illumination condition and environment. There are some problems in the application of vehicle color recognition for traffic enforcement camera system. Moreover, colors are more difficult to determine in night, so in-depth investigation on vehicle images remained to be performed in future.

\section{ACKNOWLEDGEMENTS}

The research has been supported by the natural science fund for colleges and universities in Jiangsu Province (14KJB520017), project of Jiangsu Students' innovation and entrepreneurship training (201510298028Z).

\section{REFERENCES}

[1] G Dimitrakopoulos, P. Demestichas. "Intelligent transportation systems". Vehicular Technology Magazine, IEEE, Vol. 5, 2010, pp. 77-84.

[2] R C Lee, K C Hung, H S Wang. "Real-Time Vehicle License Plate Recognition Based on Scanning and 2D Haar Discrete Wavelet Transform". Applied Mechanics and Materials, Vol 284, 2013, pp. 2402-2406.

[3] L Janowski, P KozPwski, R Baran, et al. "Quality assessment for a visual and automatic license plate recognition". Multimedia Tools and Applications. Vol 68, 2014, pp. 23-40.

[4] Y Peng, J. S Jin, S Luo, et al. "Vehicle type classification using pca with self-clustering". Multimedia and Expo Workshops, 2012 IEEE International Conference on Multimedia and Expo Workshops, 2012: 384-389.

[5] J. Wang, X. Li, H. Fan, and F. Li, "Classification of lip color based on multiple SVM-RFE," in Proc. IEEE Int. Conf. BIBMW, Nov, 2011, pp. 769-772

[6] L. Zheng, X. Li, X. Yan, F. Li, X. Zheng, and W. Li, "Lip color classification based on support vector machine and histogram". IEEE 3rd International Congress on Image and Signal Processing, Oct. 2010, pp. 1883-1886.

[7] J. W. Son, S. B. Park, and K. J. Kim, "A convolution kernel method for color recognition". IEEE Sixth International Conference on Advanced Language Processing and Web Information Technology, Aug. 2007, pp. 242-247.

[8] G. Li. J, Z. X Liu, Z. S You, et al. "Car-body color recognition algorithm based on color difference and color normalization". Computer Applications, Vol. 24, 2004, pp. 47-49.

[9] N Jing. "Research on Several Important Algorithms in Color Recognition of Vehicle-Body". Shenyang, Shenyang University of technology, 2007.

[10] K. J Kim, S. M Park, Y. J Choi. "Deciding the number of color histogram bins for vehicle color recognition". Asia-Pacific Services Computing Conference. 2008. APSCC'08. IEEE, 2008, pp, 134-138.

[11] W Yueming, H Hanwu, R Tong, et al. "Hand segmentation for augmented reality system". Digital Media and its Application in Museum \& Heritages, Second Workshop on. IEEE, 2007, pp. 395401.

[12] M. A Hearst, S. T Dumais, E Osman, et al. "Support vector machines". Intelligent Systems and their Applications, IEEE. Vol 13, 1998, pp. 18-28.

[13] V. Vapnik, "The Nature of Statistical Learning Theory". Springer New York, NY, USA, 1995.

[14] T Hastie, R Tibshirani, J Friedman, et al. "The elements of statistical learning". Springer New York, NY, USA, 2009. 